Mathematical Sciences And Applications E-Notes

Volume 3 No. 2 PP. 74-83 (2015) @ MSAEN

\title{
THE RULED SURFACES ACCORDING TO TYPE-2 BISHOP FRAME IN THE EUCLIDEAN 3-SPACE $E^{3}$
}

\author{
MELEK MASAL AND AYSSE ZEYNEP AZAK
}

(Communicated by Levent KULA)

\begin{abstract}
We have introduced the ruled surfaces which are generated from the type-2 Bishop vectors. Then, we have calculated Gaussian curvatures, mean curvatures and integral invariants of these surfaces. Also the fundamental forms, geodesic curvatures, normal curvatures and geodesic torsions are calculated and some results are obtained.
\end{abstract}

\section{INTRODUCTION}

First of all the studies with respect to the Bishop frames have been introduced by L. R. Bishop in 1975, [2]. This frame has an important role and many applications in different fields, such as Biology and Computer Graphics, $[2,3,6,7,10]$. In this area being studies of many geometers have caused to occur a new Bishop frame. Thus, Yllmaz and Turgut have introduced a new version of Bishop frame using a common vector field as binormal vector field of a regular frame and called this frame type-2 Bishop frame. Additionally, they have given the type-2 Bishop spherical indicatrices, [12]. Later, Kizıltuğ and et al have defined slant helices and obtained some characterizations of slant helices according to type-2 Bishop frame in $E^{3},[8]$. Furthermore, he has characterized the inextensible flows according this new version of Bishop frame, [9]. In [11], the author has studied the classical differential geometry of these curves according to type-2 Bishop frame.

This paper organized as follows. Firstly, we investigate the ruled surfaces which are generated from the type-2 Bishop vectors $N_{1}, N_{2}, B$. Then, we calculate Gaussian curvatures, mean curvatures and integral invariants of these surfaces. Finally, the fundamental forms, geodesic curvatures, normal curvatures and geodesic torsions are calculated and some results are given.

\section{Preliminaries}

Let $\alpha$ be a regular curve in the Euclidean 3-space. Denote by $\{T, N, B\}$ and $\left\{N_{1}, N_{2}, B\right\}$ the Frenet frame and type-2 Bishop Frame along the unit speed curve

Date: January ?, 2015 and, in revised form, June ?, 2015

2000 Mathematics Subject Classification. 53A04, 53A05.

Key words and phrases. Bishop frame, ruled surfaces, Euclidean 3-space. 
THE RULED SURFACES ACCORDING TO TYPE-2 BISHOP FRAME IN THE EUCLIDEAN 3-SPACE $\boldsymbol{E}^{3}$

$\alpha$, respectively. Then Frenet formulas and type-2 Bishop Frame are given by

$$
\begin{aligned}
& T^{\prime}=\kappa N \\
& N^{\prime}=-\kappa T+\tau B \\
& B^{\prime}=-\tau N
\end{aligned}
$$

and

$$
\begin{aligned}
& N_{1}^{\prime}=-k_{1} B \\
& N_{2}^{\prime}=-k_{2} B \\
& B^{\prime}=k_{1} N_{1}+k_{2} N_{2} .
\end{aligned}
$$

where all differentiations are with respect to the arc-length parameter $s$ of the space curve $\alpha,[5,12]$.

The relations between Frenet and type-2 Bishop frames are

$$
\left[\begin{array}{l}
T \\
N \\
B
\end{array}\right]=\left[\begin{array}{ccc}
\sin \theta(s) & -\cos \theta(s) & 0 \\
\cos \theta(s) & \sin \theta(s) & 0 \\
0 & 0 & 1
\end{array}\right]\left[\begin{array}{c}
N_{1} \\
N_{2} \\
B
\end{array}\right]
$$

On the other hand, type-2 Bishop curvatures are defined by

$$
\begin{aligned}
& k_{1}(s)=-\tau \cos \theta(s) \\
& k_{2}(s)=-\tau \sin \theta(s) .
\end{aligned}
$$

where $\theta=\arctan \left(\frac{k_{2}}{k_{1}}\right), \quad \theta^{\prime}=\kappa=\frac{\left(\frac{k_{2}}{k_{1}}\right)^{\prime}}{1+\left(\frac{k_{2}}{k_{1}}\right)^{2}}$.

In order to investigate the type-2 Bishop frame's relation with Frenet frame

$$
\begin{aligned}
& B^{\prime}=-\tau N=k_{1} N_{1}+k_{2} N_{2} \\
& T^{\prime}=\kappa N=\theta^{\prime}\left(\cos \theta N_{1}+\sin \theta N_{2}\right)
\end{aligned}
$$

and

$$
k_{1}^{2}+k_{2}^{2}=\tau^{2} .
$$

As $X$ moves along the curve $\alpha$, it generates a ruled surface $M$ given by the regular parametrization

$$
\varphi(s, v)=\alpha(s)+v X(s)
$$

where the curve $\alpha$ is called a base curve and $X$ is called the ruling of the surface $M$. The striction curve and distribution parameter of the surface $M$ are given by

$$
\bar{\alpha}(s)=\alpha(s)-\frac{\left\langle T, X^{\prime}\right\rangle}{\left\|X^{\prime}\right\|^{2}} X(s)
$$

and

$$
P_{X}=\frac{\operatorname{det}\left(T, X, X^{\prime}\right)}{\left\|X^{\prime}\right\|^{2}}
$$

respectively. If ruled surface $M$ is closed ruled surface, then Steiner translation vector is 


$$
V=\oint_{(\alpha)} d \alpha
$$

Furthermore, the pitch of $M$ is $[4,5]$

$$
l_{X}=\langle V, X\rangle .
$$

If $\varphi_{s}=\frac{\partial \varphi(s, v)}{\partial s}$ and $\varphi_{v}=\frac{\partial \varphi(s, v)}{\partial v}$, then the standart unit normal vector field of the ruled surface $M$ can be given by

$$
n=\frac{\varphi_{s} \wedge \varphi_{v}}{\left\|\varphi_{s} \wedge \varphi_{v}\right\|}
$$

The first fundamental form $I$, the second fundamental form $I I$ of the ruled surface $M$ is given by

$$
\begin{aligned}
& I=E d s^{2}+2 F d s d v+G d v^{2}, E=\left\langle\varphi_{s}, \varphi_{s}\right\rangle, \quad F=\left\langle\varphi_{s}, \varphi_{v}\right\rangle, \quad G=\left\langle\varphi_{v}, \varphi_{v}\right\rangle \\
& \text { (2.12) } \quad I I=L d s^{2}+2 M d s d v+N d v^{2}, L=\frac{\operatorname{det}\left(\varphi_{s}, \varphi_{v}, \varphi_{s}\right)}{\sqrt{E G-F^{2}}}, M=\frac{\operatorname{det}\left(\varphi_{s}, \varphi_{v}, \varphi_{s v}\right)}{\sqrt{E G-F^{2}}}, \quad N=\frac{\operatorname{det}\left(\varphi_{s}, \varphi_{v}, \varphi_{v v}\right)}{\sqrt{E G-F^{2}}}
\end{aligned}
$$

respectively. If $L N-M^{2}\langle 0$, then the ruled surface has the hyperbolic points, [1]. So the Gaussian curvature $K$ and the mean curvature $H$ of the ruled surface $M$ are given by

$$
K=\frac{L N-M^{2}}{E G-F^{2}} \quad \text { and } \quad H=\frac{L G+E N-2 M F}{2\left(E G-F^{2}\right)}
$$

respectively.

A developable surface is a surface with zero Gaussian curvature.

A surface $M \subset I R^{3}$ is minimal if and only if its mean curvature vanishes identically, $[4,5]$.

The geodesic curvature, the normal curvature and the geodesic torsion which associate the curve $\alpha(s)$ on the surface $M$ can be computed as follows

$$
\kappa_{g}=\left\langle n \wedge T, T^{\prime}\right\rangle, \kappa_{n}=\left\langle\alpha^{\prime \prime}, n\right\rangle, \tau_{g}=\left\langle n \wedge n^{\prime}, T^{\prime}\right\rangle .
$$

$\alpha$ curve is an asymptotic line of surface if and only if normal curvature $\kappa_{n}$ vanishes. $\alpha$ curve is an geodesic curve if and only if geodesic curvature $\kappa_{g}$ vanishes.

$\alpha$ curve is a principal line if and only if the geodesic torsion $\tau_{g}$ vanishes, $[1,4]$.

\section{The Ruled Surfaces According to type-2 Bishop Frame}

In this section, the ruled surfaces which are generated by the vectors $N_{1}, N_{2}, B$ according to type-2 Bishop frame will be introduced.

Let $\alpha$ be a regular curve and the set $\left\{N_{1}, N_{2}, B\right\}$ be the type-2 Bishop frame of the curve $\alpha$. Then the parametric representations of the ruled surfaces $M_{1}, M_{2}, M_{3}$ are

$$
\begin{aligned}
& \varphi(s, v)=\alpha(s)+v N_{1}(s) \\
& \zeta(s, v)=\alpha(s)+v N_{2}(s) \\
& \psi(s, v)=\alpha(s)+v B(s)
\end{aligned}
$$

and these ruled surfaces are called first rectifying surface, second rectifying surface and binormal surface according to type-2 Bishop frame, respectively. If we 
THE RULED SURFACES ACCORDING TO TYPE-2 BISHOP FRAME IN THE EUCLIDEAN 3-SPACE $\boldsymbol{E}^{2}$

calculate the differentials of the parametric representations of the ruled surfaces $M_{1}, M_{2}, M_{3}$ with respect to the parameters $s$ and $v$, we get

$$
\left\{\begin{array}{l}
\varphi_{s}=\sin \theta N_{1}-\cos \theta N_{2}-v k_{1} B, \quad \varphi_{v}=N_{1} \\
\varphi_{s s}=\left(\kappa \cos \theta-v k_{1}^{2}\right) N_{1}+\left(\kappa \sin \theta-v k_{1} k_{2}\right) N_{2} \\
\varphi_{v v}=0, \quad \varphi_{s v}=-k_{1} B
\end{array}\right.
$$

$$
\left\{\begin{array}{l}
\zeta_{s}=\sin \theta N_{1}-\cos \theta N_{2}-v k_{2} B, \quad \zeta_{v}=N_{2} \\
\zeta_{s s}=\left(\kappa \cos \theta-v k_{1} k_{2}\right) N_{1}+\left(\kappa \sin \theta-v k_{2}^{2}\right) N_{2}+\left(-k_{1} \sin \theta+k_{2} \cos \theta-v k_{2}^{\prime}\right) B \\
\zeta_{v v}=0, \quad \zeta_{s v}=-k_{2} B .
\end{array}\right.
$$

$$
\left\{\begin{array}{l}
\psi_{s}=\left(\sin \theta+v k_{1}\right) N_{1}+\left(-\cos \theta+v k_{2}\right) N_{2}, \quad \psi_{v}=B \\
\psi_{s s}=\left(\kappa \cos \theta+v k_{1}^{\prime}\right) N_{1}+\left(\kappa \sin \theta+v k^{\prime}{ }_{2}\right) N_{2}-v \tau^{2} B \\
\psi_{v v}=0, \quad \psi_{s v}=k_{1} N_{1}+k_{2} N_{2}
\end{array}\right.
$$

Hence the following determinants and norms can be expressed

$$
\begin{aligned}
& \left\{\begin{array}{l}
\operatorname{det}\left(\varphi_{s s}, \varphi_{s}, \varphi_{v}\right)=v k_{1} k_{2}\left(\frac{\kappa}{\tau}+v k_{1}\right)+v \frac{k^{\prime}{ }_{1} k_{1}}{\tau} \\
\operatorname{det}\left(\varphi_{v v}, \varphi_{s}, \varphi_{v}\right)=0, \quad \operatorname{det}\left(\varphi_{s v}, \varphi_{s}, \varphi_{v}\right)=\frac{k_{1}^{2}}{\tau}
\end{array}\right. \\
& \left\|\varphi_{s}\right\|=\sqrt{1+v^{2} k_{1}^{2}}, \quad\left\|\varphi_{v}\right\|=1 \\
& \left\{\begin{array}{l}
\operatorname{det}\left(\zeta_{s s}, \zeta_{s}, \zeta_{v}\right)=\frac{v k_{2} k_{2}^{\prime}}{{ }^{\tau}}-v k_{1} k_{2}\left(\frac{\kappa}{\tau}+v k_{2}\right), \\
\operatorname{det}\left(\zeta_{s v}, \zeta_{s}, \zeta_{v}\right)=\frac{k_{2}^{2}}{\tau}, \quad \operatorname{det}\left(\zeta_{v v}, \zeta_{s}, \zeta_{v}\right)=0
\end{array}\right. \\
& \left\|\zeta_{s}\right\|=\sqrt{1+v^{2} k_{2}^{2}}, \quad\left\|\zeta_{v}\right\|=1 \\
& \left\{\begin{array}{l}
\operatorname{det}\left(\psi_{s s}, \psi_{s}, \psi_{v}\right)=-\kappa+\frac{v}{\tau}\left(k_{1} k_{1}^{\prime}+k_{2} k_{2}^{\prime}\right)+v^{2}\left(k_{2} k_{1}^{\prime}-k_{1} k_{2}^{\prime}\right), \\
\operatorname{det}\left(\psi_{v v}, \psi_{s}, \psi_{v}\right)=0, \quad \operatorname{det}\left(\psi_{s v}, \psi_{s}, \psi_{v}\right)=\tau
\end{array}\right. \\
& \left\|\psi_{s}\right\|=\sqrt{1+v^{2} \tau^{2}}, \quad\left\|\psi_{v}\right\|=1
\end{aligned}
$$

Thus the following theorem can be given.

Theorem 3.1. The striction curves of the first rectifying surface, second rectifying surface and binormal surface according to type-2 Bishop frame are also base curves.

Proof. It is clear from the equations (2.2) and (2.7).

Theorem 3.2. The dralls of the first rectifying surface, second rectifying surface and binormal surface according to type-2 Bishop frame are

$$
P_{N_{1}}=\frac{1}{\tau}, P_{N_{2}}=\frac{1}{\tau}, P_{B}=\frac{1}{\tau}
$$

Proof. If we consider the equations (2.2), (2.4) and (2.8)

$$
P_{N_{1}}=-\frac{\cos \theta}{k_{1}}=\frac{1}{\tau}, \quad P_{N_{2}}=-\frac{\sin \theta}{k_{2}}=\frac{1}{\tau}, \quad P_{B}=\frac{1}{\tau}
$$

are obtained. This completes the proof. 
Theorem 3.3. The pitches of the first rectifying surface, second rectifying surface and binormal surface according to type-2 Bishop frame are

$$
l_{N_{1}}=\oint\left(-\frac{k_{2}}{\tau}\right) d s, l_{N_{2}}=\oint\left(\frac{k_{1}}{\tau}\right) d s, l_{B}=0
$$

respectively.

Proof. Steiner translation vector according to type-2 Bishop frame is

$$
V=\oint d \alpha=\oint T d s
$$

Considering the equation (2.3),

is found.

$$
V=\oint\left(\sin \theta N_{1}-\cos \theta N_{2}\right) d s
$$

Hence taking the inner product of the last equation with the vectors $N_{1}, N_{2}, B$ and using the equation (2.10), the following are obtained

$$
\left\{\begin{array}{l}
l_{N_{1}}=\oint \sin \theta d s \\
l_{N_{2}}=\oint(-\cos \theta) d s \\
l_{B}=0
\end{array}\right.
$$

On the other hand, if the equation (2.4) is used for the above equations, one gets:

$$
l_{N_{1}}=\oint\left(-\frac{k_{2}}{\tau}\right) d s, \quad l_{N_{2}}=\oint \frac{k_{1}}{\tau} d s, \quad l_{B}=0 .
$$

Theorem 3.4. Along the base curve of a closed ruled surface with the motion of type-2 Bishop frame $\left\{N_{1}, N_{2}, B\right\}$, the components of Steiner translation vector of this motion constitute pitches of the ruled surfaces which are generated by type-2 Bishop vectors.

Proof. Taking into consideration the proof of Theorem 3.3

$$
V=l_{N_{1}} N_{1}+l_{N_{2}} N_{2}+l_{B} B
$$

are found.

Theorem 3.5. The first fundamental forms of the ruled surfaces $M_{1}, M_{2}, M_{3}$ according to type-2 Bishop frame are

$$
\begin{aligned}
& I_{N_{1}}=\left(1+v^{2} k_{1}^{2}\right) d s^{2}-2 \frac{k_{2}}{\tau} d s d v+d v^{2} \\
& I_{N_{2}}=\left(1+v^{2} k_{2}^{2}\right) d s^{2}+2 \frac{k_{1}}{\tau} d s d v+d v^{2}
\end{aligned}
$$

and

$$
I_{B}=\left(1+v^{2} \tau^{2}\right) d s^{2}+d v^{2}
$$

Proof. If we consider the equations (2.12) and (3.2) then the coefficients of the first fundamental form for the ruled surface $M_{1}$ are

$$
E_{N_{1}}=1+v^{2} k_{1}^{2}, \quad F_{N_{1}}=-\frac{k_{2}}{\tau}, \quad G_{N_{1}}=1
$$

where

$$
E_{N_{1}} G_{N_{1}}-F_{N_{1}}^{2}=\left(\frac{k_{1}}{\tau}\right)^{2}\left(1+v^{2} \tau^{2}\right)>0
$$


THE RULED SURFACES ACCORDING TO TYPE-2 BISHOP FRAME IN THE EUCLIDEAN 3-SPACE $E$ S̊

is obtained. Thus the first fundamental form of the first rectifying surface $M_{1}$ is given by

$$
I_{N_{1}}=\left(1+v^{2} k_{1}^{2}\right) d s^{2}-2 \frac{k_{2}}{\tau} d s d v+d v^{2}
$$

Similarly, from the equations (2.12) and (3.3), we can obtain the coefficients of the ruled surface $M_{2}$ as the following

$$
E_{N_{2}}=1+v^{2} k_{2}^{2}, \quad F_{N_{2}}=\frac{k_{1}}{\tau}, \quad G_{N_{2}}=1
$$

where

$$
E_{N_{2}} G_{N_{2}}-F_{N_{2}}^{2}=\frac{k_{2}^{2}\left(1+v^{2} \tau^{2}\right)}{\tau^{2}}>0
$$

So, it is easy to see that the first fundamental form of the second rectifying surface $M_{2}$ is defined by

$$
I_{N_{2}}=\left(1+v^{2} k_{2}^{2}\right) d s^{2}+2 \frac{k_{1}}{\tau} d s d v+d v^{2}
$$

If we take the equations (2.12) and (3.4), then the coefficients of the first fundamental form of the ruled surface $M_{3}$ are given by

$$
E_{B}=1+v^{2} \tau^{2}, \quad F_{B}=0, \quad G_{B}=1
$$

where

$$
E_{B} G_{B}-F_{B}^{2}=1+v^{2} \tau^{2}>0
$$

Thus, the first fundamental form of the binormal surface is found as

$$
I_{B}=\left(1+v^{2} \tau^{2}\right) d s^{2}+d v^{2}
$$

Theorem 3.6. The second fundamental forms of the ruled surfaces $M_{1}, M_{2}, M_{3}$ according to type-2 Bishop frame are

$$
\begin{aligned}
& I I_{N_{1}}=\frac{v k_{1}^{\prime}+v k_{2}\left(\kappa+v k_{1} \tau\right)}{\sqrt{1+v^{2} \tau^{2}}} d s^{2}+\frac{2 k_{1}}{\sqrt{1+v^{2} \tau^{2}}} d s d v \\
& I I_{N_{2}}=\frac{v k_{2}^{\prime}-v k_{1}\left(\kappa+v k_{2} \tau\right)}{\sqrt{1+v^{2} \tau^{2}}} d s^{2}+\frac{2 k_{2}}{\sqrt{1+v^{2} \tau^{2}}} d s d v \\
& I I_{B}=\frac{-\kappa \tau+v\left(k_{1} k_{1}^{\prime}+k_{2} k_{2}^{\prime}\right)+v^{2} \tau\left(k_{2} k_{1}^{\prime}-k_{1} k_{2}^{\prime}\right)}{\tau \sqrt{1+v^{2} \tau^{2}}} d s^{2}+\frac{2 \tau}{\sqrt{1+v^{2} \tau^{2}}} d s d v
\end{aligned}
$$

respectively.

Proof. From equations (2.12) and (3.5), we can compute the coefficients of the second fundamental form as the following:

$$
L_{N_{1}}=\frac{v k_{1}^{\prime}+v k_{2}\left(\kappa+v k_{1} \tau\right)}{\sqrt{1+v^{2} \tau^{2}}}, \quad M_{N_{1}}=\frac{k_{1}}{\sqrt{1+v^{2} \tau^{2}}}, \quad N_{N_{1}}=0
$$

where

$$
L_{N_{1}} N_{N_{1}}-M_{N_{1}}^{2}=-\frac{k_{1}^{2}}{1+v^{2} \tau^{2}}<0
$$

Hence the second fundamental form of the first rectifying surface $M_{1}$ is

$$
I I_{N_{1}}=\frac{v k_{1}^{\prime}+v k_{2}\left(\kappa+v k_{1} \tau\right)}{\sqrt{1+v^{2} \tau^{2}}} d s^{2}+\frac{2 k_{1}}{\sqrt{1+v^{2} \tau^{2}}} d s d v
$$

Similarly, equations (2.12) and (3.7) yield

$$
L_{N_{2}}=\frac{v k_{2}^{\prime}-v k_{1}\left(\kappa+v k_{2} \tau\right)}{\sqrt{1+v^{2} \tau^{2}}}, \quad M_{N_{2}}=\frac{k_{2}}{\sqrt{1+v^{2} \tau^{2}}}, \quad N_{N_{2}}=0
$$


where

$$
L_{N_{2}} N_{N_{2}}-M_{N_{2}}^{2}=-\frac{k_{2}^{2}}{1+v^{2} \tau^{2}}<0
$$

So, the second fundamental form of the second rectifying surface $M_{2}$ takes the form:

$$
I I_{N_{2}}=\frac{v k_{2}^{\prime}-v k_{1}\left(\kappa+v k_{2} \tau\right)}{\sqrt{1+v^{2} \tau^{2}}} d s^{2}+\frac{2 k_{2}}{\sqrt{1+v^{2} \tau^{2}}} d s d v
$$

On the other hand, equations (2.12) and (3.9) lead to

$$
L_{B}=\frac{-\kappa \tau+v\left(k_{1} k_{1}^{\prime}+k_{2} k_{2}^{\prime}\right)+v^{2} \tau\left(k_{2} k_{1}^{\prime}-k_{1} k_{2}^{\prime}\right)}{\tau \sqrt{1+v^{2} \tau^{2}}}, \quad M_{B}=\frac{\tau}{\sqrt{1+v^{2} \tau^{2}}}, \quad N_{B}=0
$$

where

$$
L_{B} N_{B}-M_{B}^{2}=-\frac{\tau^{2}}{1+v^{2} \tau^{2}}<0
$$

Consequently, the second fundamental form of the binormal surface $M_{3}$ is given by

$$
I I_{B}=\frac{-\kappa \tau+v\left(k_{1} k_{1}^{\prime}+k_{2} k_{2}^{\prime}\right)+v^{2} \tau\left(k_{2} k_{1}^{\prime}-k_{1} k_{2}^{\prime}\right)}{\tau \sqrt{1+v^{2} \tau^{2}}} d s^{2}+\frac{2 \tau}{\sqrt{1+v^{2} \tau^{2}}} d s d v
$$

Result 3.1. First rectifying surface, second rectifying surface and binormal surface have hyperbolic points and two real asymptotic lines passes from these points.

Result 3.2. Let $K_{N_{1}}, K_{N_{2}}$ and $K_{B}$ denote the Gaussian curvatures and $H_{N_{1}}, H_{N_{2}}$ and $H_{B}$ denote the mean curvatures of the ruled surfaces $M_{1}, M_{2}, M_{3}$. Then the Gaussian and mean curvatures of these surfaces according to type-2 Bishop frame are

$$
K_{N_{1}}=\frac{\tau^{2}}{\left(1+v^{2} \tau^{2}\right)^{2}}, \quad K_{N_{2}}=-\frac{\tau^{2}}{k_{2}\left(1+v^{2} \tau^{2}\right)^{\frac{3}{2}}}, \quad K_{B}=-\frac{\tau^{2}}{\left(1+v^{2} \tau^{2}\right)^{2}}
$$

and

$$
\begin{aligned}
& H_{N_{1}}=\frac{k_{1} k_{2} \tau\left(2+v^{2} \tau^{2}\right)+v \tau^{2}\left(k_{1}{ }^{\prime}+k_{2} \kappa\right)}{2 k_{1}^{2}\left(1+v^{2} \tau^{2}\right)^{\frac{3}{2}}}, \quad H_{N_{2}}=\frac{-k_{1} k_{2} \tau\left(2+v^{2} \tau^{2}\right)+v \tau^{2}\left(k_{2}{ }^{\prime}-k_{1} \kappa\right)}{2 k_{2}^{2}\left(1+v^{2} \tau^{2}\right)^{\frac{3}{2}}}, \\
& H_{B}=\frac{-\kappa \tau+v\left(k_{1}{k^{\prime}}^{\prime}+k_{2} k^{\prime}{ }_{2}\right)+v^{2} \tau\left(k_{2} k^{\prime}{ }_{1}-k_{1} k^{\prime}{ }_{2}\right)}{2 \tau\left(1+v^{2} \tau^{2}\right)^{\frac{3}{2}}}
\end{aligned}
$$

respectively.

Result 3.3. First rectifying surface, second rectifying surface and binormal surface are minimal if and only if the base curves of the surface aren't planar and the following conditions are satisfied, respectively.

$$
\begin{aligned}
& k_{1} k_{2} \tau\left(2+v^{2} \tau^{2}\right)+v \tau^{2}\left(k_{1}{ }^{\prime}+k_{2} \kappa\right)=0 \\
& k_{1} k_{2} \tau\left(2+v^{2} \tau^{2}\right)-v \tau^{2}\left(k_{2}{ }^{\prime}-k_{1} \kappa\right)=0
\end{aligned}
$$

and

$$
-\kappa \tau+v\left(k_{1} k_{1}^{\prime}+k_{2} k_{2}^{\prime}\right)+v^{2} \tau\left(k_{2} k_{1}^{\prime}-k_{1} k_{2}^{\prime}\right)=0 .
$$

Result 3.4. First rectifying surface, second rectifying surface and binormal surface are developable if and only if the base curves of the surfaces aren't planar. 
THE RULED SURFACES ACCORDING TO TYPE-2 BISHOP FRAME IN THE EUCLIDEAN 3-SPACE 183

Theorem 3.7. Let $n_{N_{1}}, n_{N_{2}}$ and $n_{B}$ denote the unit normals of the surfaces $M_{1}, M_{2}$ and $M_{3}$ according to type-2 Bishop frame, respectively. These unit normals are given by

$$
\begin{aligned}
& n_{N_{1}}=-\frac{v \tau}{\sqrt{1+v^{2} \tau^{2}}} N_{2}-\frac{1}{\sqrt{1+v^{2} \tau^{2}}} B \\
& n_{N_{2}}=\frac{v \tau}{\sqrt{1+v^{2} \tau^{2}}} N_{1}-\frac{1}{\sqrt{1+v^{2} \tau^{2}}} B \\
& n_{B}=\frac{k_{1}+v k_{2} \tau}{\tau\left(1+v^{2} \tau^{2}\right)} N_{1}+\frac{k_{2}-v k_{1} \tau}{\tau\left(1+v^{2} \tau^{2}\right)} N_{2} .
\end{aligned}
$$

Proof. If we calculate the vectorial product of $\varphi_{s}$ and $\varphi_{v}$, then $\varphi_{s} \wedge \varphi_{v}$ is written as

$$
\varphi_{s} \wedge \varphi_{v}=-v k_{1} N_{2}-\frac{k_{1}}{\tau} B
$$

Considering the equation (2.11) with the above equation

$$
n_{N_{1}}=-\frac{v \tau}{\sqrt{1+v^{2} \tau^{2}}} N_{2}-\frac{1}{\sqrt{1+v^{2} \tau^{2}}} B
$$

is found. Similarly, the vectorial product of $\zeta_{s}$ and $\zeta_{v}$ is

$$
\zeta_{s} \wedge \zeta_{v}=v k_{2} N_{1}-\frac{k_{2}}{\tau} B
$$

and again from (2.11), we have

$$
n_{N_{2}}=\frac{v \tau}{\sqrt{1+v^{2} \tau^{2}}} N_{1}-\frac{1}{\sqrt{1+v^{2} \tau^{2}}} B
$$

Using equation (3.4), we obtain

$$
\psi_{s} \wedge \psi_{v}=\frac{k_{1}+v k_{2} \tau}{\tau} N_{1}+\frac{k_{2}-v k_{1} \tau}{\tau} N_{2}
$$

From equation (2.11), we have

$$
n_{B}=\frac{k_{1}+v k_{2} \tau}{\tau\left(1+v^{2} \tau^{2}\right)} N_{1}+\frac{k_{2}-v k_{1} \tau}{\tau\left(1+v^{2} \tau^{2}\right)} N_{2}
$$

Theorem 3.8. Geodesic curvatures of first rectifying surface, second rectifying surface and binormal surface according to type-2 Bishop frame are

$$
\kappa_{g_{N_{1}}}=-\frac{\kappa}{\sqrt{1+v^{2} \tau^{2}}}, \quad \kappa_{g_{N_{2}}}=-\frac{\kappa}{\sqrt{1+v^{2} \tau^{2}}}
$$

and

respectively.

$$
\kappa_{g_{B}}=0
$$

Proof. The proof is obtained from equation (2.14) and Theorem 3.7.

Result 3.5. i) The base curves of the first and second rectifying surfaces can't be geodesic curves.

ii) The base curve of the binormal surface is a geodesic curve.

Theorem 3.9. Let $\kappa_{n_{N_{1}}}, \kappa_{n_{N_{2}}}$ and $\kappa_{n_{B}}$ denote the normal curvatures of the surfaces $M_{1}, M_{2}$ and $M_{3}$ according to type-2 Bishop frame, respectively. These normal curvatures are given by

$$
\kappa_{n_{N_{1}}}=\frac{\kappa k_{2} v}{\sqrt{1+v^{2} \tau^{2}}}, \quad \kappa_{n_{N_{2}}}=-\frac{\kappa k_{1} v}{\sqrt{1+v^{2} \tau^{2}}}, \quad \kappa_{n_{B}}=-\frac{\kappa}{1+v^{2} \tau^{2}} .
$$


Proof. The proof is clear from the equation (2.14) and Theorem 3.7.

Result 3.6. i) The base curves of the first rectifying surface and second rectifying surface are asymptotic lines if and only if the torsion $\tau$ vanishes.

ii) The base curve of the binormal surface can't be an asymptotic line.

Theorem 3.10. Geodesic torsions of first rectifying surface, second rectifying surface and binormal surface according to type-2 Bishop frame are

$$
\tau_{g_{N_{1}}}=\frac{v \kappa k_{1}\left(v \tau^{2} k_{2}+\tau^{\prime}\right)}{\tau\left(1+v^{2} \tau^{2}\right)}, \quad \tau_{g_{N_{2}}}=\frac{v \kappa k_{2}\left(v \tau^{2} k_{1}+\tau^{\prime}\right)}{\tau\left(1+v^{2} \tau^{2}\right)}, \quad \tau_{g_{B}}=-\frac{v \kappa \tau^{2}}{\left(1+v^{2} \tau^{2}\right)^{2}}
$$

respectively.

Proof. Calculating the differentials of the unit normals $n_{N_{1}}, n_{N_{2}}$ and $n_{B}$ of the surfaces $M_{1}, M_{2}$ and $M_{3}$ with respect to $s$

$$
\begin{aligned}
& n_{N_{1}}^{\prime}=-\frac{k_{1}}{\sqrt{1+v^{2} \tau^{2}}} N_{1}+\left\{\left(\frac{v \tau}{\sqrt{1+v^{2} \tau^{2}}}\right)^{\prime}-\frac{k_{2}}{\sqrt{1+v^{2} \tau^{2}}}\right\} N_{2}+\left\{\frac{v k_{2} \tau}{\sqrt{1+v^{2} \tau^{2}}}-\left(\frac{1}{\sqrt{1+v^{2} \tau^{2}}}\right)^{\prime}\right\} B \\
& n_{N_{2}}^{\prime}=\left\{\left(\frac{v \tau}{\sqrt{1+v^{2} \tau^{2}}}\right)^{\prime}-\frac{k_{1}}{\sqrt{1+v^{2} \tau^{2}}}\right\} N_{1}-\frac{k_{2}}{\sqrt{1+v^{2} \tau^{2}}} N_{2}+\left\{-\frac{v k_{1} \tau}{\sqrt{1+v^{2} \tau^{2}}}-\left(\frac{1}{\sqrt{1+v^{2} \tau^{2}}}\right)^{\prime}\right\} B
\end{aligned}
$$

and

$$
n_{B}^{\prime}=\left(\frac{k_{1}+v k_{2} \tau}{\tau\left(1+v^{2} \tau^{2}\right)}\right)^{\prime} N_{1}+\left(\frac{k_{2}-v k_{1} \tau}{\tau\left(1+v^{2} \tau^{2}\right)}\right)^{\prime} N_{2}-\frac{\tau}{1+v^{2} \tau^{2}} B
$$

are found. Also from the equation (2.14), geodesic torsions of the surfaces $M_{1}, M_{2}$ and $M_{3}$ are given by as follows:

$$
\tau_{g_{N_{1}}}=\frac{v \kappa k_{1}\left(v \tau^{2} k_{2}+\tau^{\prime}\right)}{\tau\left(1+v^{2} \tau^{2}\right)}, \quad \tau_{g_{N_{2}}}=\frac{v \kappa k_{2}\left(v \tau^{2} k_{1}+\tau^{\prime}\right)}{\tau\left(1+v^{2} \tau^{2}\right)}, \quad \tau_{g_{B}}=-\frac{v \kappa \tau^{2}}{\left(1+v^{2} \tau^{2}\right)^{2}} .
$$

Result 3.7. i) The base curve of the first rectifying surface is a principal line if and only if the equality $v \tau^{2} k_{2}+\tau^{\prime}=0$ is satisfied.

ii) The base curve of the second rectifying surface is a principal line if and only if the equality $v \tau^{2} k_{1}+\tau^{\prime}=0$ is satisfied.

iii) The base curve of the binormal surface is a principal line if and only if the base curve of the surface is planar.

Result 3.8. There exists the following relationships between the geodesic curvatures, geodesic torsions and normal curvatures of the surfaces $M_{1}, M_{2}$ and $M_{3}$

and

$$
\begin{aligned}
& \tau \kappa \tau_{g_{N_{1}}}+\kappa_{n_{N_{2}}}\left(\tau^{2} \kappa_{n_{N_{1}}}-\tau^{\prime} \kappa_{g_{N_{1}}}\right)=0, \\
& \tau \kappa \tau_{g_{N_{2}}}+\kappa_{n_{N_{1}}}\left(\tau^{2} \kappa_{n_{N_{2}}}+\tau^{\prime} \kappa_{g N_{2}}\right)=0,
\end{aligned}
$$

$$
\kappa \tau_{g_{B}}+v \tau^{2} \kappa_{n_{B}}^{2}=0
$$

\section{REFERENCES}

[1] Biran, L., Diferansiyel Geometri Dersleri. İstanbul Üniversitesi Fen Fakültesi Yayınları. İstanbul, 1975.

[2] Bishop, L. R., There is more than one way to frame a curve. American Mathematical Monthly 82 (1975), no. 3, 246-251.

[3] Clauvelin N., Olson W. K., Tobias I., Characterizations of the geometry and topology of DNA pictured as a discrete collection of atoms. Journal of Chemical Theory and Computation 8 (2012), no. 3, 1092-1107. 
THE RULED SURFACES ACCORDING TO TYPE-2 BISHOP FRAME IN THE EUCLIDEAN 3-SPACE 183

[4] Do Carmo, M. P., Differential Geometry of Curves and Surfaces. Prentice Hall Inc. Englewood Cliffs. New Jersey, 1976.

[5] Hacısalihoğlu, H. Hilmi, Diferensiyel Geometri. İnönü Üniversitesi. Fen-Edebiyat Fakültesi Yayınları, Malatya, 1983.

[6] Han C. Y., Nonexistence of rational rotation-minimizing frames on cubic curves. Computer Aided Geometric Design 25 (2008), no. 4-5, 298-304.

[7] Hanson A. J., Ma H., Parallel transport approach to curve framing. Indiana University 425, vol. 11, 1995.

[8] Kızıltuğ S., Kaya S., Tarakçı Ö., The slant helices according to type-2 Bishop frame in Euclidean 3-space. International Journal of Pure and Applied Mathematics 85 (2013), no. 2, 211-222.

[9] Kızıltuğ S., On characterization of inextensible flows of curves according to type-2 Bishop frame in $E^{3}$. Mathematical and Computational Applications 19 (2014), no. 1, 69-77.

[10] Shoeemake K., Animating rotation with quaternion curves. in Proceedings of the 12th Annual Conference on Computer Graphics and Interactive Techniques, 245-254, 1985.

[11] Özyllmaz E., Classical differential geometry of curves according to type-2 Bishop trihedra. Mathematical and Computational Applications 16 (2011), no. 4, 858-867.

[12] Yilmaz S., Turgut M., A new version of Bishop frame and an application to spherical images. Journal of Mathematical Analysis and Applications 371 (2010), 764-776.

Sakarya University, Faculty of Education, Department of Elementary Education, HENDEK-54300, SAKARYA-TURKEY

E-mail address: mmasal@sakarya.edu.tr

Sakarya University, Faculty of Education, Department of Elementary Education, HENDEK-54300, SAKARYA-TURKEY

E-mail address: apirdal@sakarya.edu.tr 